\title{
Mast cell production of IL-4 and TNF may be required for protective and pathological responses in gastrointestinal helminth infection
}

\author{
MX Ierna $^{1}$, HE Scales $^{1}$, KL Saunders $^{1}$ and CE Lawrence ${ }^{1}$
}

\begin{abstract}
Expulsion of the gastrointestinal nematode Trichinella spiralis is associated with Th2 responses and intestinal inflammation, which correlate with a marked mast cell (MC) response. To address the role of MC-derived cytokines in the induction of protective responses, WBB6F1-KitW/KitW-v (W/W') mice were reconstituted with wild-type, tumor necrosis factor (TNF)- $\alpha^{-/-}$, or interleukin (IL)- $4^{-/-}$bone marrow (BM) prior to infection with $T$. spiralis. W/WN mice reconstituted with $\mathrm{TNF}-\alpha^{-/-}$or IL-4-/- BM expelled the parasite less efficiently and showed diminished enteropathy, whereas protective responses were normal in $W / W^{N}$ mice reconstituted with wild-type BM and were accompanied by intestinal pathology. MC responses were reduced in $W / W^{2}$ mice reconstituted with IL-4 ${ }^{-/-} \mathrm{BM}$ and to a lesser extent when reconstituted with TNF- $\alpha^{-/-}$. These results suggest that MC-derived IL-4 and TNF may regulate the induction of protective Th2 responses and intestinal inflammation associated with the expulsion of $T$. spiralis. Significantly, these studies suggest a role for MC-derived cytokines as autocrine growth factors.
\end{abstract}

\section{INTRODUCTION}

Potent Th2 responses concomitant with profound mastocytosis are characteristics of infection with gastrointestinal (GI) nematodes. Mast cells (MCs) have long been recognized as effector cells playing key roles in the pathology associated with inflammatory diseases ranging from allergy to GI helminth infection. Following activation, MCs contribute to inflammation by excessive and/or inappropriate release of preformed and de novo-synthesized mediators, including cytokines, which may also amplify and perpetuate responses via recruitment of inflammatory cells. ${ }^{1-8}$ Infection with GI nematode parasites, most notably Trichinella spiralis, is associated with mastocytosis accompanied by the systemic release of the $\beta$-chymase, mouse MC protease-1 (mMCP-1). ${ }^{9-11}$ Depletion of MCs or infection of MC-deficient WBB6F1-KitW/KitW-v $\left(W / W^{v}\right)$ mice has demonstrated a role for MCs in the expulsion of some GI nematode infections, ${ }^{12-15}$ with expulsion being accompanied by intestinal inflammation mediated by MCs and their products. We have previously demonstrated amelioration of the intestinal pathology accompanying the loss of $T$. spiralis following infection of $W / W^{v}$ or mMCP-1-deficient mice, ${ }^{16,17}$ and this was associated with decreased levels of interleukin (IL)-4 and tumor necrosis factor (TNF)- $\alpha$. Production of cytokines by MCs has also been shown to influence both pathological and protective immune responses. Inflammatory bowel diseases including Crohn's disease are usually associated with Th1 responses; ${ }^{18}$ however, increased numbers of MCs are present in tissue from Crohn's disease patients. ${ }^{19,20}$ Indeed, the main cell type expressing TNF- $\alpha$ in inflamed tissue of Crohn's disease are MCs. ${ }^{21,22} \mathrm{MC}$-derived TNF can act both directly and indirectly to induce intestinal inflammation, e.g., recruitment of neutrophils in bacterial peritonitis is mainly via the production of TNF- $\alpha$ by MCs. ${ }^{8}$ MC-derived TNF can also impact on the development of T-cell responses since MC-associated TNF promoted dendritic cell (DC) migration in a model of contact hypersensitivity; ${ }^{23}$ additionally, MC-derived TNF could enhance T-cell activation. ${ }^{24}$

Mast cells also have the capacity to skew the T-helper phenotype of the immune response. In addition to being the main cell type expressing IL-4 and IL-13 in asthma, ${ }^{25}$ our own studies have shown that $T h 2$ responses are reduced and Th1 responses increased in $W / W^{v}$ mice, suggesting a role for MCs in promoting and/or amplifying the Th2 responses responsible for protection and pathology. ${ }^{17}$ Similarly, infection of $W / W^{v}$ mice with Trichuris muris resulted in decreased levels of IL-4 and enhanced

${ }^{1}$ Strathclyde Institute of Pharmacy and Biomedical Sciences, University of Strathclyde, Glasgow, Scotland. Correspondence: CE Lawrence (catherine.lawrence@strath.ac.uk) 
IFN- $\gamma$ production. ${ }^{26}$ More recently, MCs have been shown to control the Th1/Th2 balance by modulating DCs. ${ }^{27}$ Thus, MCs have the capacity to bias an immune response toward a Th2 phenotype and contribute to the magnitude and perpetuation of Th2-biased responses. MCs may therefore act not only as effector cells in mediating expulsion of GI helminths but also could promote or amplify the Th2 response required for parasite loss and accompanying enteropathy.

In the current study, we asked whether MC-derived IL-4 and TNF affect the protective and pathological responses accompanying infection with GI helminths. We found that both MC-derived IL-4 and TNF were required for parasite expulsion, the development of enteropathy, and the accompanying Th2 response, thus implicating MC-derived cytokines in controlling the Th2 response in helminth infection.

\section{RESULTS}

\section{MC-derived IL-4 and TNF are required for GI helminth expulsion}

Previously, we demonstrated that MCs are required for the expulsion of the GI helminth T. spiralis and the accompanying enteropathy and this was associated with a decreased Th2 response. ${ }^{17}$ To directly evaluate the role of the MC-derived cytokines in parasite expulsion and the development of intestinal pathology, MC-deficient $W / W^{v}$ mice were reconstituted with bone marrow (BM) from wild-type, $\mathrm{IL}_{-} 4^{-/-}$, or $\mathrm{TNF}^{-/-}$mice. Mice were reconstituted with BM rather than BM-derived MCs (BMMCs) since it is reported that BMMCs fail to reconstitute the intestine and restore protective responses to GI helminthes. ${ }^{28}$ $\mathrm{BM}$ reconstitution of $W / W^{V}$ mice was determined by enumerating erythrocytes. Transfer of $5 \times 10^{5} \mathrm{BM}$ cells corrected the anemia of $W / W^{V}$ mice with a significant increase in the number of erythrocytes in $W / W^{v}$ mice-transferred BM from wild-type, IL-4 ${ }^{-1-}$, and $\mathrm{TNF}^{-1-}$ mice (on a C57Bl6 background) (Figure 1). Although $W / W^{v}$ mice are on a C57BL6 background, we wished

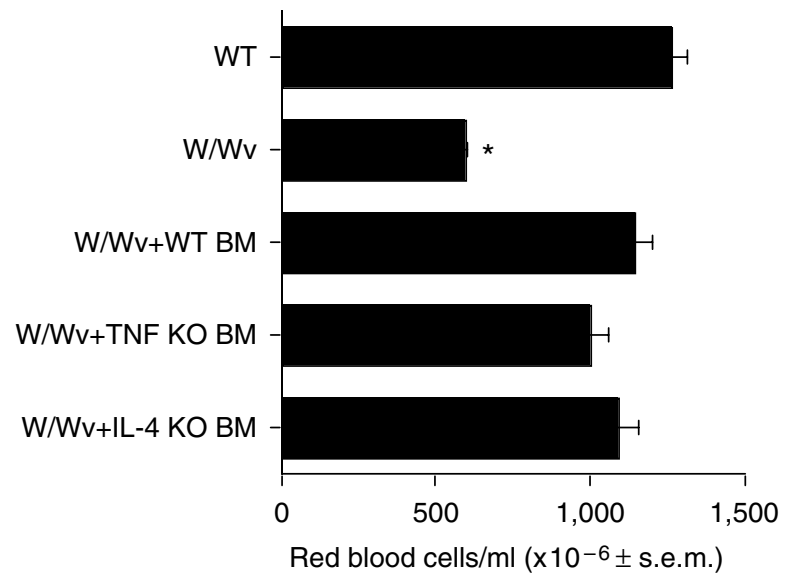

Figure 1 Bone marrow from wild-type, $\mathrm{TNF}^{-/-}$, and IL-4-/- mice corrects the anemia in $W / W^{W}$ mice. MC-deficient $W / W^{N}$ mice were reconstituted with $\mathrm{BM}$ from wild-type, $\mathrm{TNF}^{-/-}$, and IL-4-/- mice and red blood cell counts determined 8 weeks later. Data are expressed as mean number of erythrocytes $/ \mathrm{ml}+$ s.e.m., five mice were used per group. *This experiment was repeated twice with similar results; significantly different to WT $(P<0.05)$. BM, bone marrow; MC, mast cell; WT, wild-type. to ensure that the kinetics of parasite loss were similar in mice reconstituted with $\mathrm{BM}$ from homologous wild-type $W / W^{v}$ littermates and C57BL6. $W / W^{v+/+}$, C57BL6, and $W / W^{v}$ mice reconstituted with BM from $W / W^{v+l+}$ or C57BL6 mice showed similar erythrocyte number, worm expulsion, IL-4 production from antigen-stimulated mesenteric lymph nodes (MLNs), and total immunoglobin E (IgE) levels (data not shown). Thus, BM from C57BL6 was used as wild type in subsequent experiments since this was the same background as the cytokine-deficient mice used.

We have previously shown that MC-deficient mice are impaired in their ability to expel T. spiralis ${ }^{17}$ and it can be similarly seen that $W / W^{v}$ mice have significantly greater numbers of parasites present in comparison to wild-type mice at day 14 postinfection (p.i.) (Figure 2). Reconstitution of mice with BM from wild-type mice restored their ability to expel their parasites; however, $W / W^{v}$ mice reconstituted with $\mathrm{IL}_{-} 4^{-1-}$ or $\mathrm{TNF}^{-/-} \mathrm{BM}$ had significantly higher worm burdens in comparison to wildtype mice (Figure 2).

\section{MC-derived IL-4 and TNF are required for the enteropathy accompanying GI helminth infection}

Infection of mice with $T$. spiralis resulted in significant villus atrophy, crypt hyperplasia, increase in numbers of mitotic figures, and increased gut weight (Figure 3). In comparison, MC-deficient $W / W^{v}$ mice have significantly reduced levels of enteropathy with significantly longer villi, shorter crypts, fewer mitotic figures, and lower gut weight similar to that previously described. ${ }^{17}$ Reconstitution of $W / W^{v}$ mice with wild-type BM resulted in enteropathy similar to that in wild-type mice with significant villus atrophy, crypt hyperplasia, increased numbers of mitotic figures, and gut weight. In contrast, reconstitution of

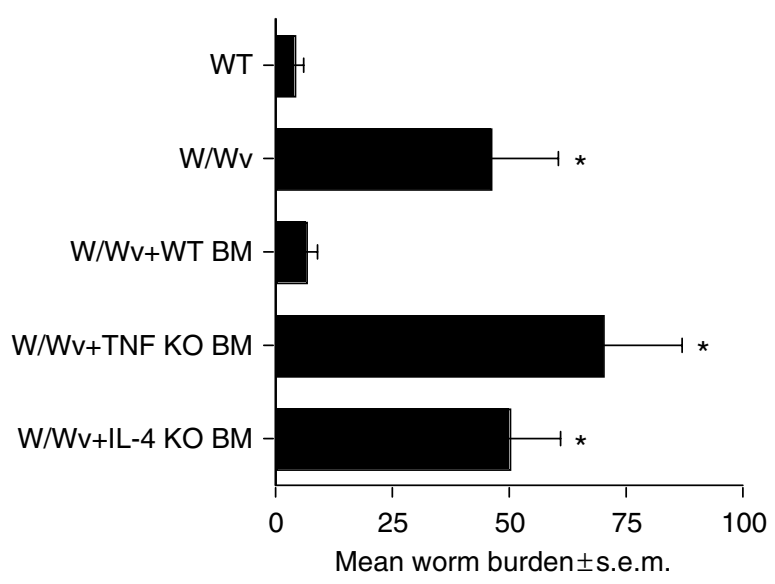

Figure 2 Parasite expulsion is delayed in MC-deficient mice reconstituted with $\mathrm{TNF}^{-/-}$or IL-4 $4^{-/-}$BM. Establishment and expulsion of $T$. spiralis was measured in wild-type and MC-deficient mice reconstituted with BM from wild-type, IL-4 $4^{-/-}$, or TNF ${ }^{-/-}$mice at days 6 and 14 p.i. The small intestine was excised and the total number of worms present was counted. Data are expressed as mean number of worms per mouse + s.e.m., five mice were used per group. *This experiment was repeated twice with similar results; significantly different to wild-type C57BL/6 mice $(P<0.05)$. BM, bone marrow; MC, mast cell; p.i., postinfection; WT, wild-type. 


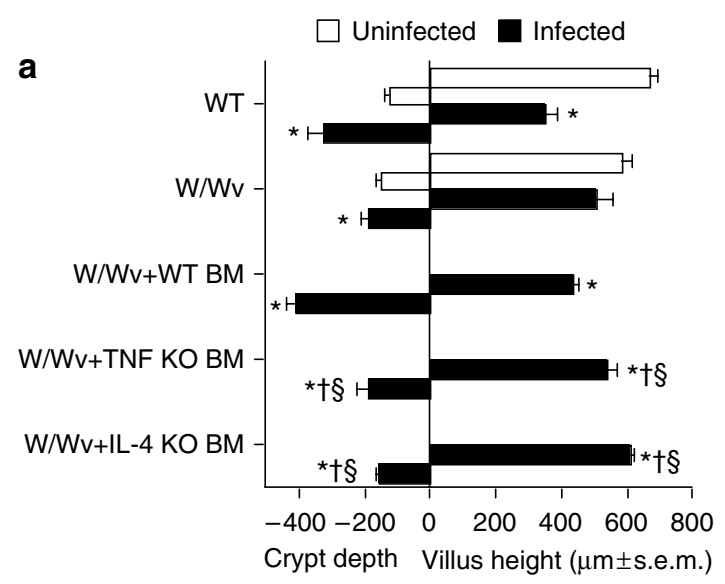

b

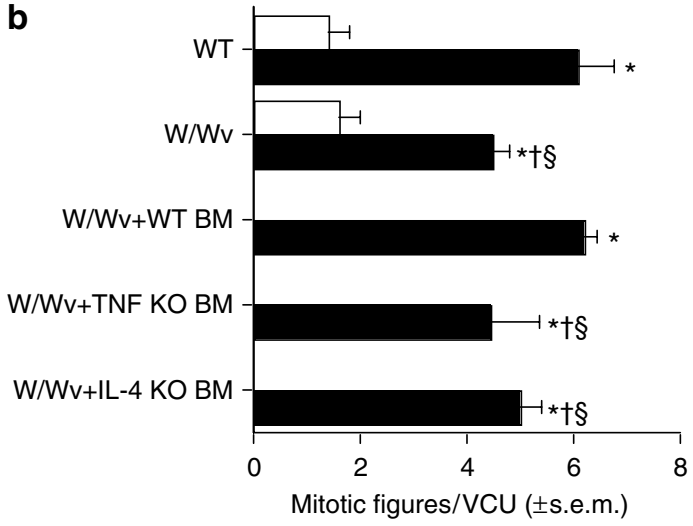

C

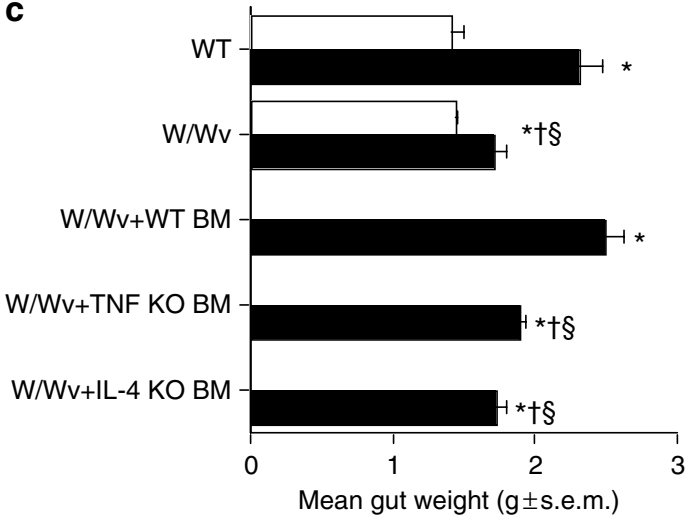

Figure 3 Parasite-induced enteropathy is reduced in MC-deficient mice reconstituted with $\mathrm{TNF}^{-/-}$or IL-4 ${ }^{-/-} \mathrm{BM}$. The development of villus atrophy and crypt hyperplasia, proliferation in crypt epithelium, and intestinal edema was measured in wild-type and MC-deficient mice

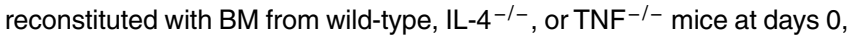
6 , and 14 p.i. Data are expressed as (a) mean length of villus and crypts $(\mu \mathrm{m})+$ s.e.m., (b) mean number of mitotic figures per villus crypt unit (MF/VCU) per mouse, or (c) mean weight (g)+s.e.m. Five mice were used per group. *This experiment was repeated twice with similar results; significantly different to uninfected mice. ${ }^{\dagger}$ Significantly different to wildtype C57BL/6 mice. $\S^{S}$ Significantly different to $W / W^{N}+\mathrm{WT}$ BM $(P<0.05)$.

$W / W^{v}$ mice with BM from either IL-4 ${ }^{-/-}$or $\mathrm{TNF}^{-/-}$mice failed to restore intestinal inflammation with significantly reduced villus atrophy, crypt hyperplasia, decreased numbers of mitotic figures, and decreased gut weight in comparison to $W / W^{v}$ mice reconstituted with wild-type BM.
MC-derived IL-4 and TNF are required for the induction of Th2 responses accompanying GI helminth infection

Mast cell-deficient $W / W^{v}$ mice have previously been shown to have reduced Th2 responses following infection with T. spiralis. ${ }^{17}$ Therefore, we wished to determine whether the presence of MC-derived cytokines could be responsible for the induction of Th2 responses accompanying infection with GI helminths. Infection of wild-type mice with $T$. spiralis resulted in a significant increase in the production of the Th2 cytokines IL-4, IL-13 and IL-9 in comparison to uninfected mice (Figure 4a-c). $W / W^{v}$ mice had significantly reduced levels of IL-4, IL-13, and IL-9 in comparison to wild-type mice, whereas reconstitution of $W / W^{v}$ mice with wild-type BM restored the levels of these cytokines similar to wild-type. In contrast, reconstitution of $W / W^{v}$ mice with IL-4 $4^{-1-}$ or TNF ${ }^{-1-}$ BM failed to restore the levels of Th2 cytokines to wild-type levels. Following infection with T. spiralis, $W / W^{v}$ mice reconstituted with wild-type BM produced similar low levels of the Th1 cytokine IFN- $\gamma$ as wild-type mice (Figure 4e), while unreconstituted $W / W^{v}$ mice and those reconstituted with IL-4 $4^{-/-}$or $\mathrm{TNF}^{-/-}$BM produced significantly higher levels than wild-type mice. Stimulation of MLN cells with ConA elicited similar levels of IL-4 in all groups thus confirming that reconstituted $W / W^{v}$ mice were competent for IL-4 production (data not shown).

We have previously shown that TNF is important for the development of enteropathy accompanying T. spiralis infection since TNF-R-deficient mice have reduced pathology. ${ }^{9}$ Evaluation of TNF- $\alpha$ levels in serum confirmed our previous findings that $W / W^{v}$ mice have reduced levels in comparison to wild-type mice following $T$. spiralis infection (Figure 4d). Reconstitution of mice with wild-type BM restored levels of TNF- $\alpha$ following infection to that similar to wild-type animals, in contrast, while levels were significantly increased in $W / W^{v}$ mice reconstituted with IL-4 $4^{-/-}$BM in comparison to unreconstituted $W / W^{v}$; this was significantly lower than those reconstituted with wild-type BM. $W / W^{v}$ mice reconstituted with $\mathrm{TNF}^{-1-} \mathrm{BM}$ showed no significant increase in serum TNF- $\alpha$ following infection with T. spiralis. This therefore suggests that MCs are an important cellular source of TNF produced following infection with GI helminths.

To further evaluate the effect on Th responses of reconstituting $W / W^{v}$ mice with BM from wild-type, $\mathrm{IL}_{-} 4^{-/-}$, or $\mathrm{TNF}^{-1-}$ mice, antibody levels were determined in serum. Measurement of total serum IgE showed an increase in levels in wild-type mice following infection, while levels were also increased following infection of $W / W^{v}$ mice; this was significantly lower than that observed in wild-type mice (Figure 5a). Reconstitution of $W / W^{v}$ mice with wild-type BM resulted in an increase in IgE levels similar to those observed in wild-type mice whereas those reconstituted with IL-4 ${ }^{-1-}$ or $\mathrm{TNF}^{-/-} \mathrm{BM}$ were comparable to that seen in $W / W^{v}$ mice. A similar pattern of responses was seen when Trichinella antigen-specific responses were measured (Figure 5b) with significantly increased IgG1 responses observed following infection of wild-type or $W / W^{v}$ mice reconstituted with wild-type BM. Responses of $W / W^{v}$ mice were increased in comparison to uninfected mice but significantly 
a
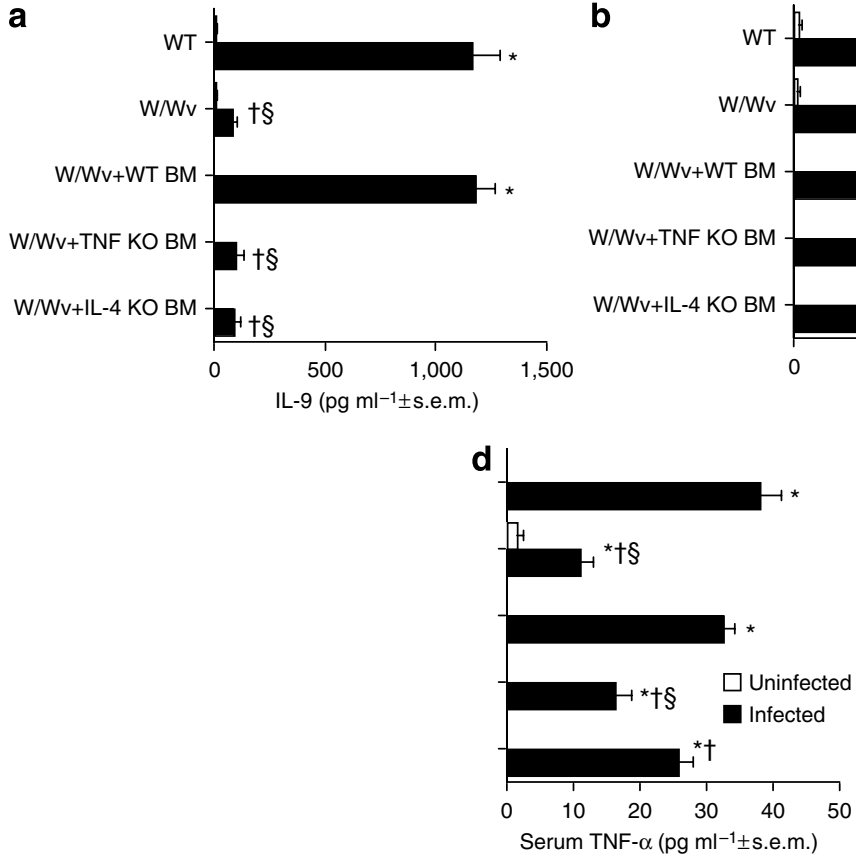

b
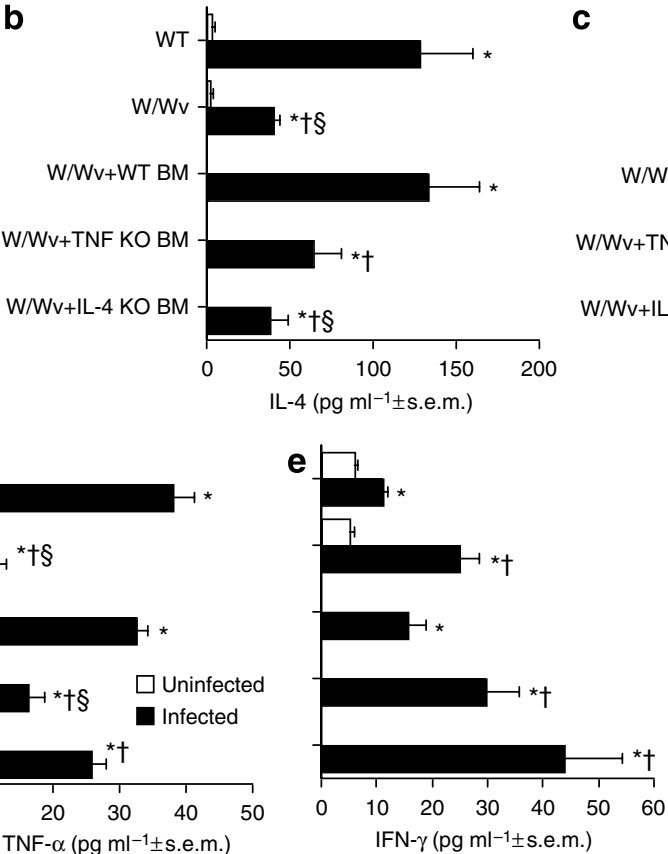

C

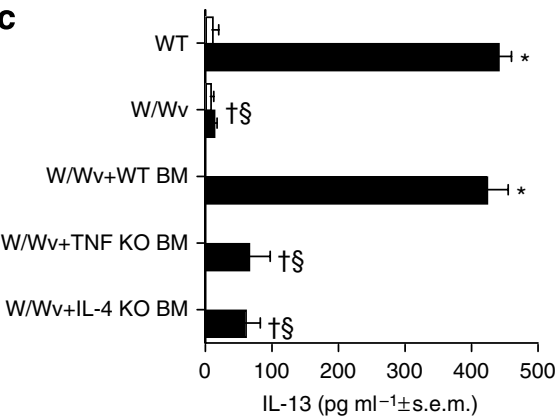

Figure 4 Th2 cytokines are reduced in MC-deficient mice reconstituted with $\mathrm{TNF}^{-/-}$or IL-4-/- BM. MLNs were removed from uninfected and infected mice, wild-type and MC-deficient mice reconstituted with BM from wild-type, IL-4 ${ }^{-1-}$, or TNF ${ }^{-1-}$ mice at day 6 p.i. and levels of (a) IL-9, (b) IL-4, (c) IL-13, and (e) IFN- $\gamma$ determined. Single-cell suspensions were made from individual animals and cultured at $1 \times 10^{6} \mathrm{cells}$ with $50 \mu \mathrm{gml}{ }^{-1} \mathrm{TAg}$ for $24 \mathrm{~h}$ and cytokine levels in culture supernatants determined by ELISA. (d) Serum levels of TNF- $\alpha$ were determined by ELISA. Data expressed mean cytokine concentration in $\mathrm{pg} \mathrm{ml}^{-1}+$ s.e.m. Five mice were used per group. ${ }^{*}$ This experiment was repeated twice with similar results; significantly different to uninfected mice. †Significantly different to wild-type C57BL/6 mice. §Significantly different to $W / W+W T$ BM $(P<0.05)$. BM, bone marrow; C, crypt; ELISA, enzyme-linked immunosorbent assay; MC, mast cell; p.i., postinfection; WT, wild-type.

lower than that observed in wild-type mice. $W / W^{v}$ mice reconstituted with IL-4 ${ }^{-1-}$ or $\mathrm{TNF}^{-1-} \mathrm{BM}$ were similar to those seen in $W / W^{v}$ mice. There were no significant Trichinella antigenspecific IgG2a responses observed in any of the groups examined (data not shown).

\section{Mastocytosis induced by infection with helminths requires the presence of MC-derived IL-4 but not TNF}

We have previously shown that mice lacking IL-4 and TNF have reduced $\mathrm{MC}$ responses in comparison to wild-type mice; thus, it was of interest to determine whether MC-derived IL- 4 or TNF altered the capacity to mount a mastocytosis. As has been demonstrated previously, ${ }^{17}$ infection of wild-type mice with T. spiralis results in a significant mastocytosis with a significant increase in the $\beta$-chymase $\mathrm{mMCP}-1$, while an absence of MCs and $\mathrm{mMCP}-1$ is observed upon infection of $W / W^{v}$ mice (Figure 6). Reconstitution of $W / W^{v}$ mice with wild-type $\mathrm{BM}$ resulted in a restoration in both the MC number and production of mMCP-1; in contrast, however, $\mathrm{MC}$ responses were significantly lower following reconstitution with both $\mathrm{TNF}^{-/-}$and IL-4 ${ }^{-/-}$ $\mathrm{BM}$, with responses in mice transferred with $\mathrm{IL}-4^{-1-}$ being significantly lower than those reconstituted with $\mathrm{TNF}^{-/-} \mathrm{BM}$. These data therefore suggest that IL-4 and, to a lesser extent, $\mathrm{TNF}$ are required for the induction of the MC response following infection with GI helminths.

Although there are a number of reports suggesting that MCs develop normally in $\mathrm{IL}_{-} 4^{-/-}$- or $\mathrm{TNF}^{-/-}$-deficient mice, ${ }^{23,24,29-31}$ the reduced numbers of MCs seen in $W / W^{v}$ mice reconstituted with $\mathrm{IL}_{-} 4^{-1-}$ or $\mathrm{TNF}^{-1-} \mathrm{BM}$ may be due to reduced numbers of $\mathrm{MC}$ precursors (MCp) in BM. Following enumeration of $\mathrm{MCp}$ in $B M$, it was observed that there were no significant differences between wild-type $(1 / 1,135 \pm 127)$, IL- $4^{-/-}(1 / 1,256 \pm 184)$, or $\mathrm{TNF}^{-1-}$ mice $(1 / 1,454 \pm 116)$, thus suggesting that the deficiency in the MC response in IL-4 $4^{-/-}$or $\mathrm{TNF}^{-/-}$is not the result of a deficiency in the numbers of MCp.

\section{DISCUSSION}

The results from this study confirm the critical role played by MCs in the expulsion of GI helminths; furthermore, it also underlines their importance in the induction of the intestinal inflammation accompanying infection with these parasites. More importantly, it highlights the role of MCs in the production of cytokines required for the generation of protective Th2 responses. The identification of the cellular sources of Th2 cytokines is of utmost importance in understanding how Th2 responses are initiated, amplified, and perpetuated in not only helminth infection but also allergic conditions such as asthma. We show here that MC-derived IL-4 makes a major contribution to the generation and amplification of Th2 responses needed for loss of GI helminths. Crucially, we also show that MC-derived TNF is a major requirement for induction of Th2 responses by GI helminth infection.

Here, we clearly demonstrate that MCs and MC-derived cytokines contribute to the induction of $\mathrm{Th} 2$ responses induced 

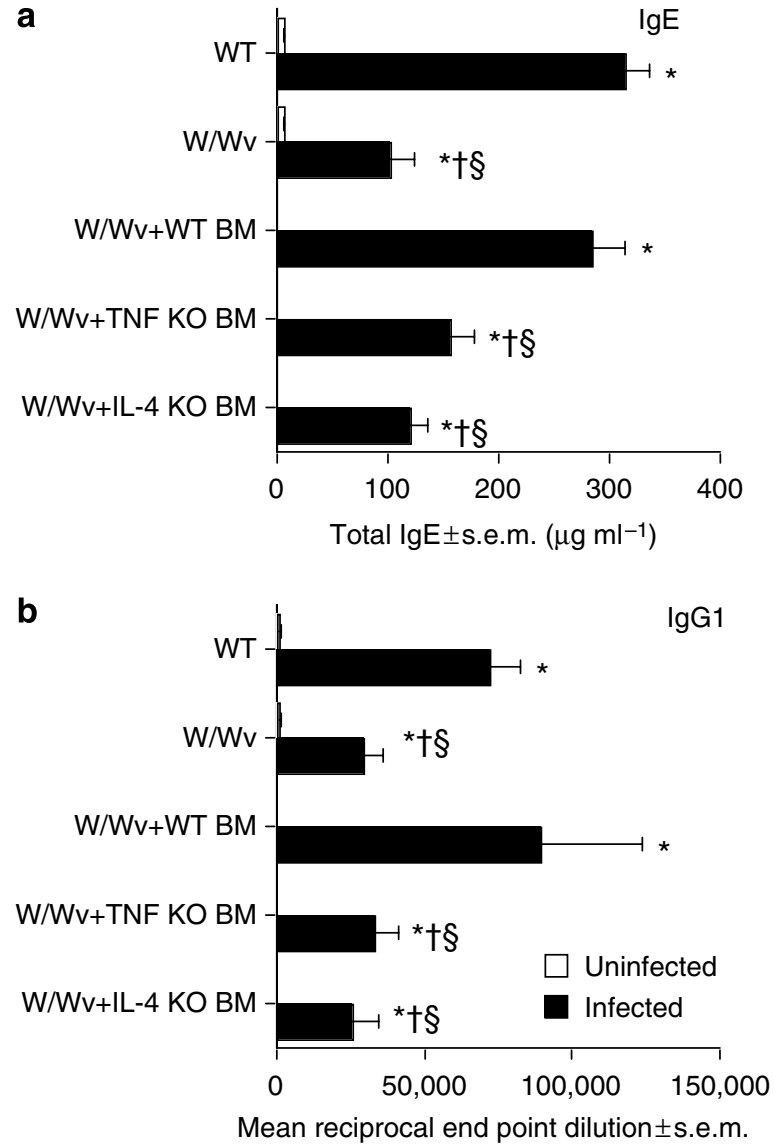

Figure 5 Total $\lg E$ and antigen-specific $\lg \mathrm{G} 1$ responses are reduced in MC-deficient mice reconstituted with $\mathrm{TNF}^{-/-}$or IL-4 ${ }^{-/}$BM. (a) Total IgE and (b) Trichinella antigen-specific IgG1 antibody levels were measured in sera from uninfected and infected wild-type and MC-deficient mice reconstituted with $\mathrm{BM}$ from wild-type, IL-4 ${ }^{-/-}$, or $\mathrm{TNF}^{-/-}$mice at day 14 p.i. Total IgE titers were measured by ELISA against a purified $\mathrm{IgE}$ standard and were expressed as mean $\mu \mathrm{g} \mathrm{ml}^{-1}+$ s.e.m. Data are expressed as mean $\mathrm{IgE}$ concentration $\left(\mu \mathrm{g} \mathrm{ml}^{-1}\right)+$ s.e.m. Antigen-specific IgG1 titers were assessed by ELISA. Plates were coated with TAg at $2 \mu \mathrm{g} \mathrm{ml}^{-1}$ and sera were serially diluted. Data expressed as reciprocal end-point dilution. Five mice were used per group. *This experiment was repeated twice with similar results; significantly different to uninfected mice. ${ }^{\dagger}$ Significantly different to wild-type C57BL/6 mice. §Significantly different to $W / W^{N}+$ WT BM $(P<0.05)$. BM, bone marrow; ELISA, enzymelinked immunosorbent assay; MC, mast cell; p.i., postinfection; WT, wild-type.

by GI nematodes and confirm our previous studies showing that MCs were required for the induction of protective and pathological responses in GI helminth infection. ${ }^{17}$ We have now extended these studies and demonstrated that loss of T. spiralis from mice reconstituted with BM from IL-4- or TNF- $\alpha$-deficient mice was reduced in comparison to mice reconstituted with $B M$ from wild-type mice, and this was associated with a reduction in Th2 responses as assessed by decreased production of the Th 2 cytokines IL- 4 and IL- 9 and the antibody isotypes IgG1 and IgE. Furthermore, the decreased Th2 responses observed in these animals were also associated with a substantially reduced mastocytosis.
MCs have been shown to modulate the capacity of DCs to polarize T cells both in vitro and in vivo. ${ }^{27}$ Degranulation of MCs leads to a decrease in tha levels of IFN- $\gamma$ and increase in the levels of IL-4 when cocultured with DCs and while it was proposed that this was mediated by histamine, the contribution of MC cytokines was not evaluated. ${ }^{27}$ Recent studies have further shown that mast cell-derived TNF can promote migration of DCs during the sensitization of acquired immune responses. Contact hypersensitivity is impaired in MC and TNF-deficient mice and this impairment was due to decreased DC migration, which could only be repaired with TNF replete MCs. MCs can also directly enhance T-cell activation and it was shown that this was mediated by MC OX40L and T cell OX40 and soluble, but not membrane-bound, TNF- $\alpha .{ }^{24}$ MCs and TNF have recently been shown to play a role in a murine model of allergic asthma; both MC-deficient $W / W^{v}$ mice and TNF-deficient mice showed reduced recruitment of inflammatory cells to the lungs and this was associated with a reduction in the local Th2 response. ${ }^{32,33}$ Furthermore, while engraftment of MC-deficient Kit ${ }^{W-s h / W-s h}$ mice with TNF-deficient BMMCs failed to restore levels of the cytokines IL-4, IL-13, IL-17 and recruitment of inflammatory cells including neutrophils and eosinophils, MCs and MC-derived TNF were not required for the induction of antigen-specific memory T cells. ${ }^{34}$ TNF-R-deficient mice or mice treated with anti-TNF- $\alpha$ have shown decreased responses to GI helminth, and this is associated with both a decrease in $\mathrm{MC}$ numbers and a decrease in Th2 responses, most notably IL-9 and IL-13. ${ }^{9,35}$ Taken together, this suggests that MC-derived TNF- $\alpha$ could play an essential role in the generation of the Th2 response essential for the expulsion of GI helminths.

The lack of a MC response as indicated by both reduced MC number and $\mathrm{mMCP}-1$ levels in the serum in mice reconstituted with BM from either IL-4- or TNF-deficient mice could be due to a number of factors including reduced MCp number, reduced recruitment to mucosal tissue, or reduced proliferation of MCp in mucosal tissue. MC precursor frequency and the ensuing mastocytosis is significantly lower in strains of micethat are poor responders to helminth infection in comparison to high-responder mouse strains. ${ }^{36}$ However, enumeration of MC precursor frequency revealed no difference between wildtype and cytokine-deficient mice and the MCp frequency we observed was similar to that reported previously for $\mathrm{C} 57 \mathrm{BL} / 6$ mice. ${ }^{36}$ Similar frequencies of MCp are observed in BM from both thymus-bearing and athymic mice; however, significant increases in MCp frequencies are detected in blood, spleen, and gut following infection of wild-type, but not athymic, mice with T. spiralis. It was concluded that this was due to a local T celldependent proliferation and maturation of residing MCp. ${ }^{37}$

Interleukin -4 has been described as being a $\mathrm{MC}$ growth factor $^{38}$ and is used to generate MCs from BM in vitro $;^{39}$ therefore, MCp from IL-4-deficient mice may lack the autocrine growthpromoting capacity of IL-4. IL-4 promotes MC proliferation and differentiation but only by synergizing with IL-3 and/or stem cell factor. ${ }^{40} \mathrm{~A}$ recent study analyzing regulation of MC IL- 4 expression by Ikaros found no difference in MC growth from IL-4deficient mice. ${ }^{31}$ Furthermore, mice deficient in IL-4 and/or 

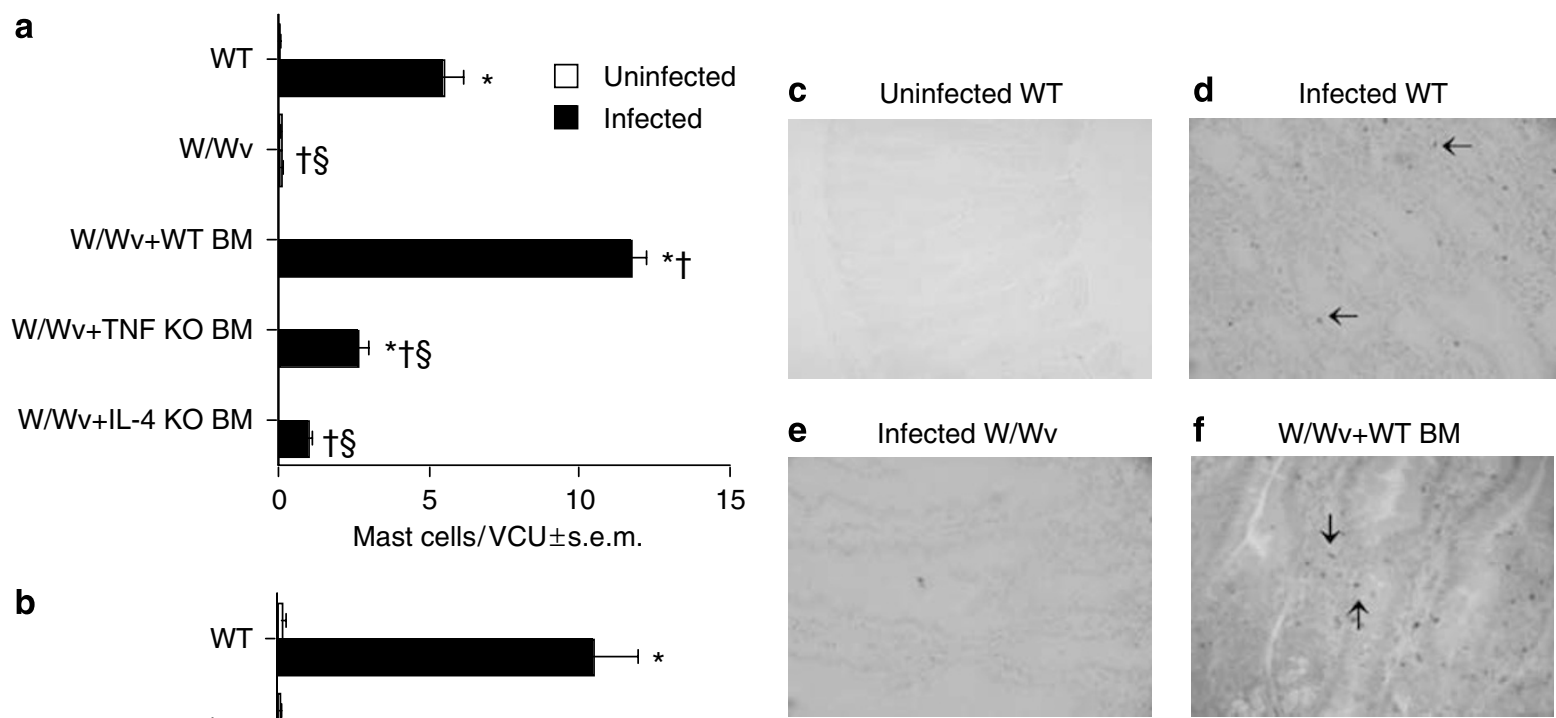

b

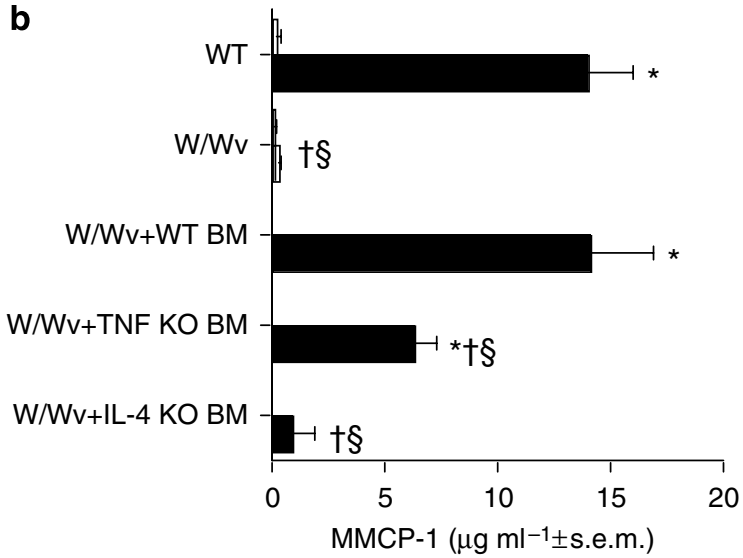

\section{g $\mathrm{W} / \mathrm{Wv}+\mathrm{IL}-4 \mathrm{KO} B M$}

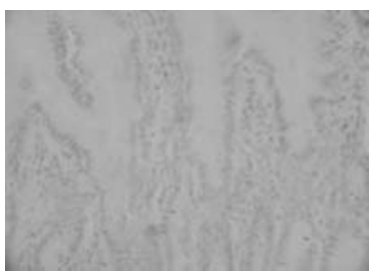

h $\quad$ W/WV+TNF KO BM

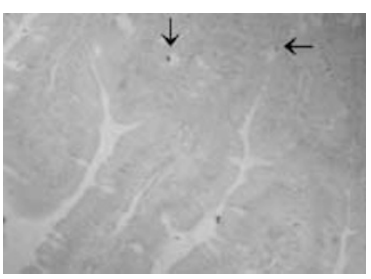

Figure 6 Mastocytosis is reduced in MC-deficient mice reconstituted with TNF $/-$ or IL-4 $4^{-/}$BM. Carnoy's fixed jejunum from uninfected and infected mice (day 14 p.i.) were processed and stained with $0.5 \%$ toluidine blue and numbers of mucosal MCs in $20 \mathrm{VCU}$ counted. Serum titers of mMCP-1 from uninfected and infected mice (day 14 p.i.) were determined by ELISA in wild-type and MC-deficient mice reconstituted with BM from wild-type, IL-4 ${ }^{-/-}$, or $\mathrm{TNF}^{-/-}$mice at day 14 p.i. Data are expressed as (a) mean number of MCs/VCU + s.e.m. or (b) mean mMCP-1 concentration $\left(\mu \mathrm{g} \mathrm{ml}{ }^{-1}\right)+$ s.e.m. Five mice were used per group. This experiment was repeated twice with similar results*; significantly different to uninfected mice. ${ }^{\dagger}$ Significantly different to wild-type C57BL/6 mice. §Significantly different to W/Wv+WT BM $(P<0.05)$. Representative photomicrographs of toluidine blue-stained intestinal tissues from wild-type and MC-deficient mice reconstituted with BM from wild-type, IL-4-/-, or TNF-/- mice at days 0 and 14 p.i. (c) uninfected wild-type, (d) infected wild-type, (e) infected $W / W v$, (f) infected $W / W v+W T$ BM, (g) infected $W / W v+$ IL-4 KO BM, (h) infected $W / W v+$ TNF KO BM. Arrow indicates MC. BM, bone marrow; ELISA, enzyme-linked immunosorbent assay; MC, mast cell; p.i., postinfection; VCU, villus per crypt units; WT, wild-type. Original magnification $\times 100$.

IL-13 have normal numbers of MCs in vivo. ${ }^{41,42}$ However, there are a number of reports in which IL-4-, IL-4-R $\alpha$-, or Stat-6-deficient mice have similar MC responses to wild-type mice. ${ }^{30,31}$ Indeed, Stat-6-deficient mice show exaggerated IL-4-induced mastocytosis, suggesting that Stat- 6 is involved in regulating MC growth. ${ }^{30}$ Other studies have shown that IL-4 induces a downregulation of key MC-signaling molecules Fc $\varepsilon$ R and c-kit ${ }^{43}$ and also promotes the apoptosis of MCs. ${ }^{44} \mathrm{TNF}-\alpha$ has not been described as a growth factor for MCs and indeed many studies have successfully cultured MCs from TNF- $\alpha$-deficient mice without any reports of decreased proliferative responses. ${ }^{23,24,29}$ Therefore, the reduced mastocytosis observed in $W / W^{v}$ mice reconstituted with TNF- $\alpha$-deficient BM does not appear to be due to reduced MCp or the proliferative capacity of precursors. The reduction in $\mathrm{MC}$ numbers seen following reconstitution of MC-deficient mice with IL-4 ${ }^{-1-}$ or $\mathrm{TNF}^{-1-} \mathrm{BM}$ may be a consequence of the reduction in the Th2 response, in particular the lack of IL-9. IL-9 has been shown to be an essential growth factor for the proliferation of mucosal MCs with IL-9-deficient mice unable to mount a mastocytosis or expel GI helminths. Taken together, this suggests that there are no basic defects in MCs from IL-4- or TNF-deficient mice and that TNF, and more importantly, IL-4 are not essential factors in promoting mastocytosis and their reduced numbers may be a reflection of the reduction in the Th2 milieu.

Critical signals for homing and recruitment of MCs to the intestine may also be provided by IL- 4 and/or TNF. MC-committed progenitors are constitutively recruited by a mechanism involving $\alpha 4 \beta 7$ integrin, an adhesion molecule also critical for T-cell homing to the gut. The ligand for this, MADCAM, is upregulated in intestinal tissue following exposure to TNF$\alpha{ }^{45}$ Recruitment of MC progenitors to the intestine requires not only adhesive interactions but also chemokine-directed migration and it has been shown that expression of chemokine 
receptors such as CXCR2 and CCR3 is critical for accumulation of MCs in mucosal tissue. ${ }^{46}$ Thus, the defects in MC populations in following reconstitution of $W / W^{v}$ mice with IL-4- and TNFdeficient BM may in general reflect the lack of a Th2 environment and upregulation of appropriate adhesion molecules and chemokines.

MC-deficient mice demonstrated significantly reduced enteropathy in comparison to wild-type mice following infection with T. spiralis and this is dependent on both MC-derived IL-4 and TNF. MC-derived TNF contributes to disease pathogenesis in a number of models of disease including sepsis, ${ }^{7,8}$ cutaneous granuloma formation, ${ }^{47}$ skin vasculitis and glomerulonephritis, ${ }^{48}$ contact hypersensitivity, ${ }^{49}$ and malaria. ${ }^{29}$ Although MCs represent a significant potential source of TNF, ${ }^{6}$ the extent to which mast cell-derived TNF might contribute directly to pathological responses in GI helminth infection is not clear. The findings that MCs and MC-derived TNF make important contributions in the development of intestinal inflammation are in accord with previous observations indicating that MCs and MC-derived TNF can promote the migration of cells involved in the inflammatory process including neutrophils and monocytes. ${ }^{8,49,50}$

In conclusion, the multidirectional MC/DC/T-cell interactions induced following GI helminth infection suggest a central role for MCs in fulfilling regulatory and/or modulatory functions affecting various aspects of the immune response and warrant further investigation. While we have not conclusively shown that MCs are the source of IL- 4 and TNF required for parasite loss, MC-derived cytokines make a major contribution to the responses induced by $T$. spiralis infection. This not only has implications for the generation of protective and pathological responses in GI helminth infections, in particular, but also other inflammatory disorders involving MCs. Thus anti-TNF therapy currently used to treat diseases such as rheumatoid arthritis and Crohn's disease could further be extended to treat diseases such as asthma in which MCs play a role due to the antagonizing effector functions of TNF from MCs.

\section{METHODS}

Animals and infection. The maintenance, infection, and recovery of T. spiralis were essentially as described previously. ${ }^{9}$ Eight- to twelveweek-old mice were infected with $400 \mathrm{~T}$. spiralis larvae on day 0 and killed at various times p.i. as detailed. Worm burdens were assessed as described previously. ${ }^{9} W / W^{v}$ mice and congenic wild-type WBB6F1- $+/+(+/+)$ mice have been well described previously ${ }^{12}$ and were obtained from Jackson Laboratories (Bar Harbor, ME). IL- $4^{-/-}$mice on a C57BL/6 background ${ }^{51}$ were originally obtained from Professor F. Brombacher, University of Cape Town, South Africa and $\mathrm{TNF}^{-/-}$mice on a C57BL/6 background were originally obtained from Professor C. Mueller, University of Bern, Switzerland. ${ }^{52}$ All mice were bred, maintained, and housed in the University of Strathclyde animal facility under standard conditions with free access to food and water and all procedures were performed in accordance with the Animals (Scientific Procedures) Act, 1986.

BM reconstitution. The method for reconstituting MC populations in $W / W^{v}$ mice by injecting BM cells of congenic mice was described previously. ${ }^{53}$ Briefly, donor wild-type, $\mathrm{IL}_{-} 4^{-/-}$, and $\mathrm{TNF}^{-/-}$mice were euthanized by cervical dislocation, and BM was flushed from their femurs using Hanks' balanced salt solution. The BM cells were then pooled, washed, and counted using trypan blue exclusion to assess viability. Recipient
$W / W^{v}$ mice were then reconstituted by tail vein injection of $5 \times 10^{6}$ viable $\mathrm{BM}$ cells suspended in sterile phosphate-buffered saline. BM-reconstituted $W / W^{v}$ mice were studied $8-12$ weeks later. Successful reconstitution was confirmed by enumerating red blood cells in reconstituted mice that revealed the correction of the anemia suffered by $W / W^{V}$ mice.

Quantitation of intestinal pathology. Mucosal architecture and epithelial-cell mitotic activity were assessed in samples of jejunum taken $10 \mathrm{~cm}$ from the pylorus, as described previously. ${ }^{9}$ Samples were fixed in $25 \%$ acetic acid $/ 75 \%$ ethanol and stained with Schiff's reagent (Sigma, Poole, UK). Specimens were microdissected and villus and crypt lengths measured using an eyepiece micrometer. The number of mitotic figures per crypt were also counted. Ten villi and crypts were measured for each sample.

MC precursor assay. A limiting dilution assay for MCp frequency was used to determine differences in MCp frequency in wild-type, IL- $4^{-1-}$, and $\mathrm{TNF}^{-1-}$ mice as previously described. ${ }^{36}$ Briefly, single-cell suspensions of $\mathrm{BM}$ cells were prepared and $1 \times 10^{7} \mathrm{BM}$ cells were serially diluted in TI3S medium (Dulbecco's modified Eagle's medium containing $10 \%$ fetal calf serum, $100 \mathrm{U} \mathrm{ml}^{-1}$ penicillin, $100 \mathrm{mg} \mathrm{ml}^{-1}$ streptomycin, $2.5 \mathrm{mg} \mathrm{ml}^{-1}$ fungizone, $2 \mathrm{mM} \mathrm{L}$-glutamine, $1 \mathrm{mM}$ sodium pyruvate (all from Gibco, Paisley, UK), $1 \mathrm{ng} \mathrm{ml}^{-1}$ recombinant human TGF-b1 (Sigma), $1 \mathrm{ng} \mathrm{ml}^{-1}$ recombinant mouse IL-3 (R\&D Systems, Abingdon, UK), $5 \mathrm{ng} \mathrm{ml}^{-1}$ recombinant mouse IL-9 (R\&D Systems), $50 \mathrm{ng} \mathrm{ml}^{-1}$ recombinant mouse SCF (Peprotech EC Ltd, London, UK)). Sixteen aliquots of $100 \mu \mathrm{l}$ of each dilution were added to a flat-bottomed 96well plate and cultured for 7 days in a humidified $5 \% \mathrm{CO}_{2}$ incubator; after feeding with fresh TI3S, medium plates were cultured for a further 7 days and then stored at $-70^{\circ} \mathrm{C}$. Plates were then freeze-thawed three times and the entire content of each well removed for quantitation of mMCP-1 levels. Numbers of MCp present were then calculated using L-Calc software.

MC quantitation. Consecutive samples of jejunum were sampled and fixed in Carnoy's fixative followed by processing using standard histological techniques. Sections were stained with $0.5 \%$ toluidine blue (Sigma) in $0.5 \mathrm{M} \mathrm{HCl}$ for $\mathrm{MC}$ visualization and counterstained with $0.5 \%$ safranine $O$ (Sigma) for 2 min. ${ }^{9}$ The number of mucosal MCs were counted in 10 villus per crypt units (VCU) and data expressed a mean number of mucosal MCs per VCU.

MMCP-1 analysis. The mMCP-1 sandwich enzyme-linked immunosorbent assay (ELISA) kit was a kind gift from Professor Hugh Miller, University of Edinburgh, Edinburgh. ${ }^{16}$

Lymphocyte culture and cytokine responses. Single-cell suspensions of MLNs were prepared and incubated in the presence of T. spiralis larval homogenate $\left(50 \mu \mathrm{g} \mathrm{ml}^{-1}\right.$ protein). Culture supernatants were harvested after $24 \mathrm{~h}$ and IL- 4 , IL-9, IL-13, and IFN- $\gamma$ levels measured by ELISA using paired antibodies as described previously. ${ }^{9}$ TNF- $\alpha$ levels were determined in serum by ELISA according to the manufacturer's instructions (PharMingen, Oxford, UK).

Measurement of antibody responses. Parasite-specific IgG1 and IgG2a and total IgE levels were determined as described previously. ${ }^{9}$ T. spiralis larval homogenate was used as a target antigen at $10 \mu \mathrm{g} \mathrm{ml}^{-1}$ in ELISA, and sera were diluted (1/100). IgG1 and IgG2a were detected using biotinylated anti-mouse IgG1 or IgG2a (PharMingen) at $2 \mu \mathrm{g} \mathrm{ml}^{-1}$ each, followed by streptavidin-peroxidase (SAPU, Carluke, UK). Total serum IgE levels were measured by ELISA, anti-mouse IgE was used as capture antibody and IgE detected using biotinylated anti-mouse IgE (PharMingen). An IgE mAb specific for trinitrophenyl (TNP) (PharMingen) was used as standard.

Statistics. Results are expressed as means \pm 1 s.e.m. Statistical differences $(P \leqslant 0.05)$ between experimental groups were determined using the Mann-Whitney $U$-test for nonparametric data. 


\section{ACKNOWLEDGMENTS}

We would like to thank Professor Frank Brombacher, University of Cape Town, South Africa for the provision of the breeding pairs of the IL-4-deficient mice. We would also like to thank Professor Hugh Miller, University of Edinburgh, Edinburgh for advice regarding $\mathrm{MC}$ precursor assay and for provision of mMCP-1 ELISA reagents. This study was supported by Wellcome Trust Grants 055503 and 62264 to CEL.

\section{DISCLOSURE}

The authors declare no conflict of interest.

\section{() 2008 Society for Mucosal Immunology}

\section{REFERENCES}

1. Wedemeyer, J., Tsai, M. \& Galli, S.J. Roles of mast cells and basophils in innate and acquired immunity. Curr. Opin. Immunol. 12, 624-631 (2000).

2. Aoki, l. et al. Contribution of mast cells to the Thelper 2 response induced by simultaneous subcutaneous and oral immunization. Immunology 98 , 519-524 (1999).

3. Lorentz, A., Schwengberg, S., Sellge, G., Manns, M.P. \& Bischoff, S.C. Human intestinal mast cells are capable of producing different cytokine profiles: role of IgE receptor cross-linking and IL-4. J. Immunol. 164, 43-48 (2000).

4. Toru, H., Pawankar, R., Ra, C., Yata, J. \& Nakahata, T. Human mast cells produce IL-13 by high-affinity IgE receptor cross-linking: enhanced IL-13 production by IL-4-primed human mast cells. J. Allergy Clin. Immunol. 102, 491-502 (1998).

5. Burd, P.R. et al. Interleukin 3-dependent and -independent mast cells stimulated with lgE and antigen express multiple cytokines. J. Exp. Med. 170, 245-257 (1989).

6. Gordon, J.R. \& Galli, S.J. Mast cells as a source of both preformed and immunologically inducible TNF-alpha/cachectin. Nature 346, 274-276 (1990).

7. Echtenacher, B., Mannel, D.N. \& Hultner, L. Critical protective role of mast cells in a model of acute septic peritonitis. Nature 381, 75-77 (1996).

8. Malaviya, R., Ikeda, T., Ross, E. \& Abraham, S.N. Mast cell modulation of neutrophil influx and bacterial clearance at sites of infection through TNF-alpha. Nature 381, 77-80 (1996).

9. Lawrence, C.E. et al. IL-4-regulated enteropathy in an intestinal nematode infection. Eur. J. Immunol. 28, 2672-2684 (1998).

10. Woodbury, R.G. et al. Mucosal mast cells are functionally active during spontaneous expulsion of intestinal nematode infections in the rat. Nature 312, 450-542 (1984).

11. Wastling, J.M., Scudamore, C.L., Thornton, E.M., Newlands, G.F. \& Miller, H.R. Constitutive expression of mouse mast cell protease-1 in normal BALB/c mice and its up-regulation during intestinal nematode infection. Immunology 90, 308-313 (1997).

12. Ha, T.Y., Reed, N.D. \& Crowle, P.K. Delayed expulsion of adult Trichinella spiralis by mast cell-deficient W/Wv mice. Infect. Immun. 41, 445-447 (1983).

13. Donaldson, L.E., Schmitt, E., Huntley, J.F., Newlands, G.F. \& Grencis, R.K A critical role for stem cell factor and c-kit in host protective immunity to an intestinal helminth. Int. Immunol. 8, 559-567 (1996).

14. Grencis, R.K., Else, K.J., Huntley, J.F. \& Nishikawa, S.I. The in vivo role of stem cell factor (c-kit ligand) on mastocytosis and host protective immunity to the intestinal nematode Trichinella spiralis in mice. Parasite Immunol. 15, 55-59 (1993).

15. Khan, A.I., Horii, Y., Tiuria, R., Sato, Y. \& Nawa, Y. Mucosal mast cells and the expulsive mechanisms of mice against Strongyloides venezuelensis. Int. J. Parasitol. 23, 551-555 (1993).

16. Knight, P.A., Wright, S.H., Lawrence, C.E., Paterson, Y.Y. \& Miller, H.R. Delayed expulsion of the nematode Trichinella spiralis in mice lacking the mucosal mast cell-specific granule chymase, mouse mast cell protease-1. J. Exp. Med. 192, 1849-1856 (2000).

17. Lawrence, C.E., Paterson, Y.Y., Wright, S.H., Knight, P.A. \& Miller, H.R. Mouse mast cell protease- 1 is required for the enteropathy induced by gastrointestinal helminth infection in the mouse. Gastroenterology 127 , 155-165 (2004).

18. Blumberg, R.S., Saubermann, L.J. \& Strober, W. Animal models of mucosal inflammation and their relation to human inflammatory bowel disease. Curr. Opin. Immunol. 11, 548-656 (1999).
19. Nolte, H., Spjeldnaes, N., Kruse, A. \& Windelborg, B. Histamine release from gut mast cells from patients with inflammatory bowel diseases. Gut 31, 791-794 (1990).

20. King, T., Biddle, W., Bhatia, P., Moore, J. \& Miner, P.B.J. Colonic mucosal mast cell distribution at line of demarcation of active ulcerative colitis. Dig. Dis. Sci. 37, 490-495 (1992).

21. Lilja, I., Gustafson-Svard, C., Franzen, L. \& Sjodahl, R. Tumor necrosis factor-alpha in ileal mast cells in patients with Crohn's disease. Digestion 61, 68-76 (2000).

22. Bischoff, S.C. et al. Mast cells are an important cellular source of tumour necrosis factor alpha in human intestinal tissue. Gut 44, 643-652 (1999).

23. Suto, $\mathrm{H}$. et al. Mast cell-associated TNF promotes dendritic cell migration. J. Immunol. 176, 4102-4112 (2006).

24. Nakae, S. et al. Mast cells enhance T cell activation: importance of mast cell costimulatory molecules and secreted TNF. J. Immunol. 176, 2238-2248 (2006)

25. Brightling, C.E. et al. Interleukin-4 and -13 expression is co-localized to mast cells within the airway smooth muscle in asthma. Clin. Exp. Allergy 33, 1711-1716 (2003).

26. Koyama, K. \& Ito, Y. Mucosal mast cell responses are not required for protection against infection with the murine nematode parasite Trichuris muris. Parasite Immunol. 22, 21-28 (2000).

27. Mazzoni, A., Siraganian, R.P., Leifer, C.A. \& Segal, D.M. Dendritic cell modulation by mast cells controls the Th1/Th2 balance in responding T cells. J. Immunol. 177, 3577-3581 (2006).

28. Abe, T. \& Nawa, Y. Localization of mucosal mast cells in W/Wv mice after reconstitution with bone marrow cells or cultured mast cells, and its relation to the protective capacity to Strongyloides ratti infection. Parasite Immunol. 9, 477-485 (1987).

29. Furuta, T., Kikuchi, T., Iwakura, Y. \& Watanabe, N. Protective roles of mast cells and mast cell-derived TNF in murine malaria. J. Immunol. 177, 3294-3302 (2006)

30. Urban, JF Jr et al. IL-13, IL-4Ralpha, and Stat6 are required for the expulsion of the gastrointestinal nematode parasite Nippostrongylus brasiliensis. Immunity 8, 255-264 (1998).

31. Gregory, G.D., Raju, S.S., Winandy, S. \& Brown, M.A. Mast cell IL-4 expression is regulated by lkaros and influences encephalitogenic Th1 responses in EAE. J. Clin. Invest. 116, 1327-1336 (2006).

32. Kim, Y.S. et al. Mast cells play a key role in the development of late airway hyperresponsiveness through TNF-alpha in a murine model of asthma. Eur. J. Immunol. 37, 1107-1115 (2007).

33. Nakae, S. et al. TNF can contribute to multiple features of ovalbumininduced allergic inflammation of the airways in mice. J. Allergy Clin. Immunol. 119, 680-686 (2007).

34. Nakae, S. et al. Mast cell-derived TNF contributes to airway hyperreactivity, inflammation, and $\mathrm{T}(\mathrm{H}) 2$ cytokine production in an asthma model in mice. J. Allergy Clin. Immunol. 120, 48-55 (2007).

35. Artis, D. et al. Tumor necrosis factor alpha is a critical component of interleukin 13- mediated protective T helper cell type 2 responses during helminth infection. J. Exp. Med. 190, 953-962 (1999).

36. Brown, J.K., Donaldson, D.S., Wright, S.H. \& Miller, H.R. Mucosal mast cells and nematode infection: strain-specific differences in mast cell precursor frequency revisited. J. Helminthol. 77, 155-161 (2003).

37. Parmentier, H.K., Teppema, J.S., van Loveren, H., Tas, J. \& Ruitenberg, E.J. Effect of a Trichinella spiralis infection on the distribution of mast cell precursors in tissues of thymus-bearing and non-thymus-bearing (nude) mice determined by an in vitro assay. Immunology 60, 565-571 (1987).

38. Madden, K.B. et al. Antibodies to IL-3 and IL-4 suppress helminthinduced intestinal mastocytosis. J. Immunol. 147, 1387-1391 (1991).

39. Tsuji, K. et al. Effects of interleukin-3 and interleukin-4 on the development of "connective tissue-type" mast cells: interleukin-3 supports their survival and interleukin-4 triggers and supports their proliferation synergistically with interleukin-3. Blood 75, 421-427 (1990).

40. Williams, C.M. \& Galli, S.J. Mast cells can amplify airway reactivity and features of chronic inflammation in an asthma model in mice. J. Exp. Med. 192, 455-462 (2000).

41. Chen, X.J., Lycke, N. \& Enerback, L. Surface and gene expression of immunoglobulin $E$ receptors on mast cells and mast-cell numbers in interleukin-4-gene knockout mice. Immunology 96, 544-550 (1999).

42. Fish, S.C., Donaldson, D.D., Goldman, S.J., Williams, C.M. \& Kasaian, M.T. IgE generation and mast cell effector function in mice deficient in IL-4 and IL-13. J. Immunol. 174, 7716-7724 (2005). 
43. Ryan, J.J. et al. IL-4 inhibits mouse mast cell Fc epsilonRI expression through a STAT6-dependent mechanism. J. Immunol. 161, 6915-6923 (1998).

44. Hu, Z.Q., Zhao, W.H., Shimamura, T. \& Galli, S.J. Interleukin-4-triggered, STAT6-dependent production of a factor that induces mouse mast cell apoptosis. Eur. J. Immunol. 36, 1275-1284 (2006).

45. Connor, E.M., Eppihimer, M.J., Morise, Z., Granger, D.N. \& Grisham, M.B. Expression of mucosal addressin cell adhesion molecule-1 (MAdCAM-1) in acute and chronic inflammation. J. Leukoc. Biol. 65, 349-355 (1999).

46. Abonia, J.P. et al. Constitutive homing of mast cell progenitors to the intestine depends on autologous expression of the chemokine receptor CXCR2. Blood 105, 4308-4313 (2005).

47. von Stebut, E., Metz, M., Milon, G., Knop, J. \& Maurer, M. Early macrophage influx to sites of cutaneous granuloma formation is dependent on MIP-1alpha/beta released from neutrophils recruited by mast cell-derived TNFalpha. Blood 101, 210-215 (2003).

48. Watanabe, N. et al. Mast cells induce autoantibody-mediated vasculitis syndrome through tumor necrosis factor production upon triggering Fcgamma receptors. Blood 94, 3855-3863 (1999).
49. Biedermann, T. et al. Mast cells control neutrophil recruitment during $T$ cell-mediated delayed-type hypersensitivity reactions through tumor necrosis factor and macrophage inflammatory protein 2. J. Exp. Med. 192, 1441-1452 (2000).

50. McLachlan, J.B. et al. Mast cell-derived tumor necrosis factor induces hypertrophy of draining lymph nodes during infection. Nat. Immunol. 4, 1199-1205 (2003).

51. Nickdel, M.B. et al. Intestinal pathology during acute toxoplasmosis is IL-4 dependent and unrelated to parasite burden. Parasite Immunol. 26, 75-82 (2004).

52. Corazza, N., Eichenberger, S., Eugster, H.P. \& Mueller, C. Nonlymphocyte-derived tumor necrosis factor is required for induction of colitis in recombination activating gene (RAG) $2\left(^{-/-}\right)$mice upon transfer of CD4(+)CD45RB(hi) T cells. J. Exp. Med. 190, 1479-1492 (1999).

53. Perdue, M.H., Masson, S., Wershil, B.K. \& Galli, S.J. Role of mast cells in ion transport abnormalities associated with intestinal anaphylaxis. Correction of the diminished secretory response in genetically mast celldeficient $W / W v$ mice by bone marrow transplantation. J. Clin. Invest. 87, 687-693 (1991). 